\title{
Multilinguales
}

15 | 2021

Recherches algériennes en sociolinguistique et en littérature : discours scientifique/discours militant, quelles frontières?

\section{Les insultes « mots-doux » dans les échanges conversationnels dans l'espace public en Côte d'Ivoire}

"Gentle- words" insults in Ivorian public conversation talk

\section{Kouakou Kouman Fodjo}

\section{(2) OpenEdition}

\section{Journals}

Édition électronique

URL : https://journals.openedition.org/multilinguales/6309

DOI : $10.4000 /$ multilinguales.6309

ISSN : 2335-1853

\section{Éditeur}

Université Abderrahmane Mira - Bejaia

\section{Référence électronique}

Kouakou Kouman Fodjo, «Les insultes « mots-doux » dans les échanges conversationnels dans l'espace public en Côte d'Ivoire », Multilinguales [En ligne], 15 | 2021, mis en ligne le 15 juin 2021, consulté le 15 février 2023. URL : http://journals.openedition.org/multilinguales/6309; DOI : https:// doi.org/10.4000/multilinguales.6309

Ce document a été généré automatiquement le 14 février 2022.

\section{cc) $(1) \ominus$}

Creative Commons - Attribution - Pas d'Utilisation Commerciale - Pas de Modification 4.0 International - CC BY-NC-ND 4.0

https://creativecommons.org/licenses/by-nc-nd/4.0/ 


\title{
Les insultes « mots-doux » dans les échanges conversationnels dans l'espace public en Côte d'Ivoire
}

\author{
"Gentle- words" insults in Ivorian public conversation talk
}

\author{
Kouakou Kouman Fodjo
}

1 L'appellation "violence verbale » fédère un ensemble de termes qui se rapportent à la violence exprimée par des mots, des expressions des cris acrimonieux, irrévérencieux et « infériorisants » à travers divers faits et actes de langage. Ainsi, non seulement ces faits et actes affectent la personne ou les groupes qui les subissent mais ils peuvent porter atteinte à leur intégrité psychologique. Au nombre de ces termes figure l' "insulte» qui est inhérente à nos pratiques sociales ainsi qu'à nos rituels conversationnels ou communicationnels. Pour cela même, l'insulte englobe divers faits et actes vu qu'elle porte sur "toute parole, toute attitude ou allusion à contenu symbolique » (J. Dubois et al., $2012: 250$ ) perçues comme dévalorisantes et blessantes.

2 Toutefois, loin d'être placée au cœur des « conflits » langagiers et donc d'être toujours envisagée dans sa dimension vexatoire, l'insulte, du fait de son importance dans la dynamique sociale des échanges conversationnels, peut s'entrevoir sous les angles de concorde et d'harmonie. Suivant ce qui précède, l'on se demande comment des mots et / ou expressions, reconnus comme « insultes » à bien des égards et qui, dans bien de circonstances, ont donné leur preuve de charge vexatoire, ne déclenchent pas systématiquement le processus d'humiliation ou de réclusion dans une posture peu gratifiante. Mieux, pourquoi le résultat débouche sur le contraire, avec des insultes qui se font «mots-doux»? Nous nous proposons d'étudier cet état de fait suivant la méthode d'analyse du discours, notamment la praxématique qui est centrée sur l'analyse de la production du sens en langage.

3 Nous nous appuyons sur un corpus composite constitué de conversations, promouvant des insultes qui fonctionnent comme des «mots-doux", entre deux catégories de personnes. La première catégorie concerne des personnes qu'unissent globalement quelque lien d'amitié, de camaraderie ou de proximité du fait de la communauté 
professionnelle ou de lieu de travail. Dans cette catégorie, l'on relève majoritairement des jeunes, conducteurs et apprentis de véhicules de transport en commun, élèves du secondaire et étudiants. L'on y note également, mais très rarement, des personnes âgées ou très âgées, peut-être par exigence sociale. En effet, fors les cas de pacte d'autorisation "d'insultes non épigrammatiques" dans le cadre des alliances interethniques, il est rare que des personnes adultes se distribuent des insultes «affectives " publiquement. Le respect et la courtoisie régulent les rapports publics entre eux et avec les autres. La seconde catégorie ressortit naturellement à des alliés ${ }^{1}$ interethniques sans distinction d'âge.

4 En termes de subdivision, notre analyse comporte trois parties. La première, "de l'insulte à l'insulte "mot-doux" ", évacue les questions théoriques en présentant les concepts d' "insulte » et d' "insulte mot-doux ». La seconde partie, "catégorisation des insultes-mots doux », relève les différentes propriétés des « insultes mots-doux ». La dernière partie, «valeurs des insultes mots-doux » aborde la question de la portée des « fausses insultes ».

\section{De l'insulte à l'insulte « mot-doux »}

5 Il s'agira ici de présenter au moins succinctement les notions de "insulte " et de «insulte mot-doux» en les définissant brièvement. Par ailleurs, nous relèverons comment et à quel moment l'« insulte » devient « insulte mot-doux ».

\subsection{De l'insulte}

6 L' " insulte » désigne l'action d'offenser, de blesser, à l'oral et de manière accidentelle ou intentionnelle. Elle garde son sens primitif d'«attaque», puisqu'elle est «une attaque verbale ». Elle s'applique aussi bien à toute parole qu'à tout acte qui sont des outrages ou dont le but poursuivi est d'outrager, de blesser la dignité ou l'honneur de leur destinataire. Reposant sur l'interaction verbale, son domaine d'application est vaste. S'il fallait en administrer la preuve, la prodigalité des termes et expressions qui la désignent et /ou avec lesquels ce mot entretient quelque relation de synonymie y suffirait assurément. En effet, l'insulte est connexe à des notions qui désignent aussi bien des actes que des comportements verbaux usuellement distincts. Ces notions sont l' " injure ", l' " offense ", le "blasphème ", le "sacrilège ", l' " invective » auxquelles on peut ajouter les "sacres" du québécois parlé ainsi que la «joute lexicale", l' « imprécation », la « mise en boite ». Il existe bien entendu une distinction d'intensité entre l'insulte et certaines de ces notions. Par ailleurs, les valeurs en contexte des unes et des autres ainsi que leurs caractéristiques pragmatiques ne sont pas à dépriser.

7 L'insulte relève par conséquent et avant tout de la violence verbale, étant elle-même une de ses multiples formes. Elle est un acte menaçant pour la face de celui qui la subit puisqu'elle induit un jugement de valeur dépréciatif. Autrement dit, l'insulte laisse des traces "psychologiques» souvent profondes et indélébiles. Elle fait intervenir a minima un objet et deux acteurs ou groupes d'acteurs. L'objet représente le terme dont fait usage l'être qui insulte. Ce terme, selon Laforest et Vincent, associe souvent

"la personne visée à des animaux connotés négativement ou à des objets ou substances perçus comme dégoûtants » (Laforest et Vincent, $2004: 60$ ),

à travers une comparaison, une métaphore, une métonymie ou bien une hyperbole. 

proposé par Lezou (Lezou, 2012) et suivant le modèle de Larguèche à propos de l'« injure » (Larguèche, 1983: 1). Sur cette base, l'insulteur est celui qui insulte; l'insultaire celui qui subit l'insulte « interpellative ", notamment dans les interactions; l'insulté désigne l'être à qui s'adresse l'insulte mais dans une relation triangulaire. Somme toute, l'insulte est un phénomène social, acte ou parole par lesquels un insulteur adjuge $\mathrm{au}(\mathrm{x})$ récipiendaire(s) des propriétés nouvelles mais négatives et dévalorisantes. Elle intervient dans un contexte de conflit, de frustration, de mécontentement. L'insulteur y a recours pour non seulement manifester son état psychologique rembruni, mais aussi et surtout pour abâtardir l'insultaire ou l'insulté. De ce point de vue, l'insulte fonctionne comme un instrument de brimade et de torture attentatoire à la dignité et au moral de l'insultaire ou de l'insulté. Le but ultime d'un tel acte réside dans la volonté de l'insulteur de les rabaisser ou de les blesser. L'insulte, telle que présentée jusqu'ici, a besoin de la validation de l'insulté ou de l'insultaire qui l'acceptent et encaissent le coup qu'elle porte. Si ces derniers ne la valident pas, l'insulte devient vaine, stérile puisqu'elle n'aura pas atteint son but. De là l'importance du concept « insulte mot-doux ».

\subsection{De l'insulte « mot-doux »}

Le groupe nominal écrit en deux mots "mot» et "doux" sans le trait d'union, permettra de spécifier ce que l'on devra entendre par «mot» et «doux» dans un premier temps avant de l'entrevoir comme concept. En effet, après avoir défini et présenté l'insulte, il parait évident de dire un mot sur la seconde composante du groupe « insulte mot-doux » pour faire ressortir l'opposition qui les caractérise et qui, en même temps, donne toute son importance et son originalité à notre étude. Suivant cela, le lexème mot est à considérer ici aux divers sens de "unité lexicale ou vocable " ou comme une litote signifiant "petit nombre de paroles, de phrases ou propos». Quant à l'adjectif "doux», il signifie selon le contexte, "affectueux, non violent». Ainsi, par le groupe nominal «mot doux», il faut entendre, "parole ou propos non violents, non agressifs » ou " parole, propos affectueux ».

Le mot composé, "mot-doux », permet de marquer qu'il existe un lien lexical étroit entre ces deux termes et en fait un concept. Ainsi, le désormais concept « mot-doux » a un sens spécifique certes, mais dont le rapport avec les sens individuels de chaque mot qui le compose reste alambiqué. Ce sens "spécial» est «affectueux, factice» et leurs synonymes respectifs en contexte. La définition même de "mot-doux » ne s'affranchit pas de la définition de l' "insulte» qu'il faut prendre à rebours. Avec cette orthographe, les groupes nominaux «insulte » et «mot-doux » forment un oxymore car leur rapprochement crée un contraste. L'« insulte mot-doux » signifie « une insulte, et donc en réalité attentatoire à la dignité et reconnue comme vexatoire mais, qui, paradoxalement, produit l'effet inverse ». Tout bien considéré, l' « insulte mot-doux " est une "fausse insulte», "un simulacre d'insulte» ou mieux une "moquerie aimante ».

11 Dans sa forme, elle ne diffère pas de l'insulte de manière générale, mais dans son fonctionnement, en contexte, elle s'oppose radicalement à celui de l'insulte. L'insulte mot-doux est une insulte détournée en une adresse affectueuse aux frontières nébuleuses avec l'insulte. La tension entre la signification ratifiée en langue, et qui est 
bien évidemment dépréciative, de l'insulte et celle actualisée et «valorisante » du mot doux reste latente. Par voie de conséquence, la menace de basculer de l'insulte motdoux à l'insulte est réelle. Tout est lié à l'état d'esprit ou à la prédisposition des acteurs de la conversation et surtout au contexte dans sa diversité (le cotexte, situation d'énonciation et les participants à la conversation) qui compte énormément dans le calcul sémantique de l'insulte mot-doux. L'insulte mot-doux, quoique mot ou expression infériorisants et turpides, procède d'une resémantisation à polarité positive et, contrairement à l'insulte, n'outrage pas, dans le contexte où elle s'utilise. C'est dire que hors de ce contexte, singulièrement dans un environnement propice à l'insulte, l'insulte mot-doux redevient outrageante et donc explicitement une insulte.

\section{Catégorisation des insultes « mots- doux »}

Par catégorisation des insultes "mots doux", il sera question de relever les constituants insultes mots-doux, d'analyser leurs propriétés linguistiques mais aussi discursives. Le corpus recueilli auprès du public provient de conversations. Les énoncés sont par conséquent oraux à l'origine. Nous les avons donc transcrits pour les besoins de l'analyse.

\subsection{Description grammaticale et énonciative des constituants « insultes mots-doux »}

13 Cette description porte sur les unités lexicales les plus utilisées pour adresser des insultes. Ce sont l'adjectif qualificatif, le nom et le groupe nominal. Les adjectifs qualificatifs constituent l'une des unités lexicales les plus sollicitées comme insultes mots-doux. Ce sont en général des adjectifs descriptifs à polarité négative ou péjorative ou dont le sens est dévalorisant, dépréciatif. Ils qualifient un nom ou un groupe nominal qui désignent une partie ou un aspect du corps humain. Les énoncés suivants l'illustrent.

- Tes fesses dures avec ton petit nez et ton vilain visage.

- C'est cela que mon mari aime. Toi, regarde ta vilaine barbe !

- Tu es un faux type, un vrai faux type!

14 Les adjectifs « vilain » ainsi que sa forme féminine « vilaine » et « faux » employés en ( 1 , 2 et 3) appartiennent au vocabulaire dépréciatif. Quant à « dures » et " petit » en (1a), ils ne sont en réalité pas nécessairement négatifs ou dévalorisants, mais leur emploi en contexte leur confère ces valeurs. Syntaxiquement, ils sont antéposés ou postposés aux noms qu'ils qualifient et sont alors épithètes :

- nom + adjectif (fesses dures en (1));

- adjectif + Nom (petit nez, vilain visage, vilain visage, faux type en (1,2 et 3)).

Ces adjectifs donnent une image "négative » et subjective des portraits physiques ainsi faits, a priori. Toutefois, ils n'outragent effectivement pas leurs destinataires. Ici, même si la qualification avilissante, bien que subjective, est avérée, elle reste inoffensive, non méchante. Elle s'accepte comme telle. Celui / celle qui la profère n'y a pas mis une méchante intention, celle de blesser ou de choquer par exemple. De cette manière celui / celle à qui elle s'adresse ne se sent nullement insulté, rabaissé. A contrario, il /elle plaisante même avec ce qui est supposé offensant, infamant. L'ambiance et le contexte 
conviviaux d'une gare de transport en commun ou d'un environnement professionnel ou d'un lieu de conversation entre jeunes apprentis ou étudiants y conduisent.

L'on rencontre également des adjectifs insultes mots-doux adressées ou autoadressées :

-4. Il / elle ne sait pas que tu es bête! Montre lui que tu es bête!

-5. Laisse-la, je vais lui montrer que je suis très bête, très sauvage !

-6. Les autorités savent que nous sommes bêtes...

-7. Vous êtes sauvages, vous-là.

-8. Tu es trop fou, tu es bête hein!

Sans aucun doute, les adjectifs «bête(s) », "sauvage(s) » " fou » ont généralement un sens avilissant. Cependant, l'avilissement est désactivé ici au profit d'une resémantisation, d'une "recharge " positive et affectueuse de ces adjectifs. Il n'est nullement question du sens dénoté des adjectifs «bête(s) », "sauvage(s) » ou «fou », affublés ou non d'adverbes d'intensité " très » ou " trop », dans ces conversations entre étudiants. Ces mêmes adjectifs se rencontrent dans des conversations entre les autres catégories sociales ou professionnelles ciblées par notre étude. Ils qualifient le moral, le comportement, l'état ou l'être. Selon le contexte, ils signifient «pouvoir faire mieux ou pire...» $(4,5,6$ et 7$)$ et « être drôle » (8).

Du point de vue syntaxique, ils apparaissent dans des constructions où ils sont attributs du sujet. Le sujet est généralement un pronom de la première et/ou de la deuxième personne, puisque nous avons des énoncés conversationnels, le dialogue est toujours latent. L'usage de la première personne, «je » (5) et «nous» (6), prévaut justement lorsque le locuteur semble se «flageller ». Quant à la deuxième personne, elle s'utilise pour les insultes mots-doux directement adressées à un allocutaire apostrophé ou représenté (4 et 7). Ils s'accompagnent souvent d'adverbes d'intensité. L'auxiliaire « être » utilisé de manière presqu'exclusive est au présent simple de l'indicatif, suivant la syntaxe :

- $\circ \mathrm{Je} /$ nous + être (présent de l'indicatif) + adjectif;

- $\mathrm{Tu} /$ vous + être (présent de l'indicatif) + adjectif.

Cette structure est modifiée par l'adverbe d'intensité qui se place après « être ».

Il arrive que lors de la conversation, l'allocutaire, quoique présent est désigné par la troisième personne. Le pronom personnel est ainsi décalé ou substitué.

-9. Ces mecs, ils sont malades hein!

-10. Lui, il n'est vraiment pas normal !

-11. Les Guérés (Gouros) sont sauvages ou les Yacouba (Sénoufo) sont bêtes...

Ainsi, le groupe nominal «ces mecs » désigne en réalité «vous» (9) alors que «il» renvoie à « tu ». Sans le changement du pronom, on aurait « vous êtes malades hein ! » ou «tu n'es pas normal». Les énoncés $(9,10$ et 11$)$ ainsi que leurs formes en structure profonde sont des insultes mots-doux. Deux d'entre eux ont le sens de "Ces mecs, ils sont (très) intelligents, habiles! » pour en vérité dire "Vous êtes très intelligents, habiles!» (9), «Lui, il est (très) intelligent, habile!" pour dire "Tu es (très) intelligent, habile!» (10). Quant à (11), il est le prototype même de l'insulte mot-doux entre alliés interethniques. " Guéré » et "Gouro », " Yacouba » et "Sénoufo » sont des groupes ethniques ivoiriens alliés. Il n'y a rien de méchant ou de frustrant dans les adjectifs utilisés. C'est juste une manière de plaisanter, pour montrer la solidité de l'alliance, l'impossibilité de violence, quelle qu'elle soit, entre les individus appartenant à ces groupes. 

adverbe de négation pour lui conférer un sens dépréciatif, disqualificatif. La structure syntaxique s'adapte avec l'inclusion des nouveaux constituants et l'exclusion de ceux qui n'y figurent plus :

- $\circ \mathrm{GN} /$ ils / il + être (présent de l'indicatif) + adjectif;

- GN/ils / il + adverbe de négation+ être (présent de l'indicatif) + adjectif (valorisant).

Enfin, l'adjectif peut être employé seul dans des énoncés du genre: «Sauvage! Bête !... ».

21 Hormis l'adjectif, le nom, déterminé ou non, motivé ou non, est également utilisé comme insulte mot-doux. Il désigne une condition de vie ou un lien (esclave, sujet), des animaux (chien, cochon, cafard...), un état (sauvage, bête), des êtres humains (père, mère, pygmée...), des personnages (Kirikou), et la liste ne semble pas close. Les noms négativement connotés s'accompagnent de déterminants possessifs, définis ou indéfinis suivant diverses structures syntaxiques :

- $\circ$ adj. possessif (ou article défini, indéfini) + nom (péjoratif ou dépréciatif) $(12,13)$;

- GN + être (présent de l'indicatif) + adj. possessif (ou article défini, indéfini) + nom (péjoratif ou dépréciatif).

-12. Mon esclave adoré ! Mon esclave!

-13. L'esclave, que fais-tu ici ? Un esclave, tu as fini ton boulot?

-14. Les baoulés (Agni, Abron) sont (des, mes, nos) esclaves, sujets.

-15. Les Guérés (Gouro) sont des sauvages.

-16. Yacouba ce n'est pas l'homme, ce sont des animaux.

Nous avons ici des exemples d'insultes mots-doux entre alliés interethniques. Ils ne se disent qu'en présence de celui à qui l'insulte mot-doux s'adresse ou d'un «représentant» du groupe auquel il appartient. Les noms, bien que "agressifs» se déchargent de leur "agressivité » et se perçoivent comme mots non violents. Il y a ici une déconsidération de la charge dévalorisante qui se transforme en un lien d'union sacrée qui transcende n'importe quelle violence. Généralement, le destinataire en rit et y répond avec les mêmes insultes mots-doux, puis l'échange se poursuit dans une bonne ambiance. La liste n'est pas exhaustive et l'on rencontre également des noms à valeur toujours rabaissante désignant des parties d'animaux (gueule, pattes...) ou des noms d'animaux comme en (17 et 18), avec le même fonctionnement.

-17. Chien! Cafard ! Cochon !...

-18. Quand je vois la gueule de mon voisin... ta gueule là je sais que tu vas manger poulet.

Dans cette catégorie d'insultes mots-doux, on observe le déterminant zéro pour les noms d'animaux (17). De plus, l'insulte mot-doux peut être constituée d'un seul nom d'animal ou d'une succession souvent rapide de noms d'animaux. La structure de (17) n'est pas pour autant figée car ces noms se rencontrent dans des structures du genre «Tu es un chien, un cafard, un cochon!». L'article défini ou l'adjectif possessif de la deuxième personne s'emploient pour "gueule » et par ricochet les insultes mots-doux similaires. (18) contient une métaphore avilissante certes, mais elle n'est pas méchante encore moins motivée, qui renvoie à la « bouche ».

Les noms se rapportant aux parents ne sont pas en marge des insultes mots-doux. L'on rencontre des énoncés du genre "Ta mère, ton père, ta grand-mère, ton grandpère » (19), qui visitent toute la généalogie, ou presque, avec un accent sur les grandsparents. L'intonation reste essentielle car le destinateur insiste sur les voyelles 
accentuées finales en tirant également sur le « $\mathrm{r}$ ». Sur cette même base, les parties du corps humain ne sont pas oubliées. Elles s'utilisent également comme insultes motsdoux. Cela donne «Ta tête, ton ventre, tes fesses... » (20). Parfois, elles visitent même les parties intimes pour donner des insultes mots-doux grossières comme «Ta pine, ton con, mon con" (21) à la fois gênantes et choquantes pour les non habitués. Des sousentendus se rattachent à ces désignations vulgaires des sexes masculin et féminin qui s'adressent indistinctement, dans de nombreux cas, aux hommes comme aux dames. Dans de tels usages, elles fonctionnent comme des mots vides, de simples plaisanteries. La structure se présente comme suit :

- $\circ$ Déterminant possessif ( $2^{\grave{e}}$ personne) + nom (de généalogie ou partie du corps humain).

Comme on peut le constater, contrairement à bien d'insultes mots-doux rencontrées jusqu'ici, qui sont en réalité de vraies insultes du fait de leur sens péjoratif, infamant, vexatoire, celles qui précèdent n'ont de coloration «méprisante » que dans la prosodie et dans le processus d'énonciation. Mais le principe étant le même, elles demeurent "inoffensives». Bien des insultes mots-doux se forment par usage concomitant du nom, de l'adjectif dans un mélange dont le modèle est " Toi-même imbécile, vaurien, enfoiré, bête, cornard ... !» (22).

\subsection{Registre de langue des insultes mots-doux}

Le type de corpus choisi, c'est-à-dire la conversation entre catégories sociales ou professionnelles, même s'il n'exclut pas les registres soutenu ou courant, il impose déjà tacitement un registre de langue, celui de la conversation. En effet, la conversation impose moins de contraintes aussi bien lexicales que grammaticales. Par ailleurs, certains des auteurs des énoncés du corpus sont peu ou pas lettrés. Sur cette base, on ne s'étonne pas que la grande majorité des insultes mots-doux empruntent un style oral qui promeut les niveaux de langue courant et, à un degré moindre, familier, voire relâché. L'adverbe «là », dans "Maudit-la! Salaud-là !» (23), ainsi que dans certains énoncés du corpus, termine certaines insultes mots-doux. Au demeurant, le vocabulaire reste essentiellement familier, souvent vulgaire. Ainsi, les adjectifs, les noms ou groupes nominaux n'appartiennent pas au vocabulaire châtié. Le nouchi achève de convaincre du registre relâché. De nombreuses insultes mots-doux foisonnent en nouchi, sorte d'argot crypté ivoirien qui est un mélange de français et de plusieurs langues du pays. La structure syntaxique ainsi que le fonctionnement énonciatif respectant ce qui est décrit jusqu'ici, nous nous contenterons de donner le sens de l'expression nouchi entre parenthèses.

-24. Malo-là ! (Malhonnête).

-25. Djandjou-là tu fais quoi ici ? (Coureur de jupons, prostitué(e)).

-26. Espèce de djaouli! kpakpato! (badaud, indiscret, importun).

-27. Apprenti vogo-là ! (vagabond).

-28. Blakoro-là ! (très bizarre).

-29. Espèce de côcô ! (tapeur, parasite).

- 30. Imbécile, "ibièkissè"2 ${ }^{2}$ idjou ! (injure grossière qui désigne le sexe de la femme).

Le registre familier, voire relâché utilisé dans les insultes mots-doux fait perdre la virulence et la rigidité des propos. Ainsi, il participe du «désarmement» des constituants naturellement ou contextuellement violents. 


\subsection{Quelques procédés stylistiques des insultes mots-doux}

L'insulte mot-doux innove dans le processus de sa mise en œuvre. Elle exploite des procédés tels la comparaison, la métaphore, à l'instar de l'insulte. Elle joue également sur les sonorités. La comparaison consiste à rapprocher un terme ou un ensemble de termes, d'un terme ou d'un ensemble de termes. Dans les insultes mots-doux, elle procède du rapprochement de deux objets ou réalités antagonistes. Ce rapprochement crée une sorte de hiatus. La comparaison débouche sur quelque chose d'abject, d'inconcevable ou d'inacceptable. L'énoncé « Regardez-le on dirait "la" » (31) est une comparaison ignominieuse. Ici, entendons qu'un être de sexe masculin, représenté ici par « $\boldsymbol{l} \boldsymbol{e}$ », se comporte comme une femme, représentée par « $\boldsymbol{l a}$ ». Ainsi, elle fonctionne comme une insulte et, prise à rebours, elle est insulte mot-doux. Sur ce modèle, on peut avoir plusieurs insultes mots-doux qui procèdent de la comparaison des parties ou aspects du corps humain, du comportement..., qui procèdent à des comparants de sorte que le résultat soit avilissant.

De même, la métaphore, avec laquelle elle partage certains traits de fonctionnement, assimile un comparé à un comparant. Les insultes mots-doux résultent également de cette figure d'analogie. La métaphore est la figure essentielle des insultes mots-doux. Elle est in praesentia dans les énoncés (4 à $8,11,12,14$ à 16$)$ où elle assimile des êtres humains à des "sauvages » et in absentia dans (17 et 18) où les humains sont désignés du nom d'animaux. On assiste à une "dépersonnification" de la personne. Quelle qu'elle soit la métaphore est dégradante. Enfin, les insultes mots-doux exploitent des procédés novateurs. L'on exploite la proximité des sonorités pour créer quelque chose de « négatif ou dégradant ». En voici quelques exemples.

-32. Le faux djo ou le faux des djos.

-33. Arbron (petit arbre).

-34. C'est nous les faux.

Il s'agit en réalité dans ces énoncés de noms de personnes et de groupes ethniques. Les noms sont «Fodjo » (32) et «Abron» (33), «Sénoufo » (34). «Fodjo» est un nom de l'ethnie "Abron ou Brong ou Bron", peuple du nord-est de la Côte d'Ivoire allié du peuple « Sénoufo » du nord. Dans les insultes mots-doux qu'ils se distribuent du fait de leur alliance, certains en arrivent à exploiter les sons des noms pour donner (32, 33 et 34). Il faut plutôt entendre « Fodjo », en (32) ; « Abron », en (« 33) ; « Sénoufo » en (34). Le résultat fait donc de "Fodjo » le faux (fo-) des « djo » ou le faux (fo-) « djo », de «Abron » arbron, entendu " arbuste » et sur le modèle de « Fodjo » on aura c'est (sé-) nous (nou-) les faux (fo). L'objectif de « rabaisser » amicalement l'allié est atteint dans tous les cas et l'on savoure ces insultes mots-doux.

\subsection{Typologies des insultes mots-doux}

Telles qu'elles définies, et suivant la typologie des insultes, les insultes mots doux se classent en ethnotype ou tribale, en personnelle et en sexotype. L'ethnotype ou encore tribale tient à ce que l'insulte mot-doux porte sur l'origine de provenance de la personne à qui elle s'adresse. C'est le cas dans (11, 14 à 16). Ici, la dimension de l'appartenance géographique ou de la race n'apparait pas. Tout se passe entre groupes ethniques alliés, chaque groupe se "croyant » supérieur à l'autre. Dans les exemples 
supra, le destinateur de l'insulte mot-doux confirme son appartenance au «bon» groupe en mettant à distance le groupe de l'autre, perçu comme « sauvage, inférieur ».

Personnelle, l'insulte mot-doux porte sur l'identité du destinataire, son estime de soi ainsi que son physique. Les énoncés 12, 13, 18, 19, 20, 25, 29 en sont des illustrations. L'insulte mot-doux porte sur les traits physiques ou des traits de comportement ainsi que sa fierté, sa dignité. Le sexotype est le type d'insulte mot-doux qui visite les parties intimes et porte sur l'orientation sexuelle du destinataire. Les exemples foisonnent avec des désignations ubuesques aussi bien en français que dans des langues ivoiriennes. On peut relever (21 et 30).

En définitive, avec les insultes mots-doux, les constituants, le registre ainsi que les procédés stylistiques utilisés visent à créer quelque chose de dévalorisant pour le destinataire de l'insulte mot-doux que ce dernier devra en retour recevoir en inversant le sens ou en le valorisant. Face à ce qu'on pourrait appeler " escalade de la violence " qui, en réalité, n'en est pas une, le spectateur non averti des réalités ivoiriennes et notamment des affinités entre les destinateurs et les destinataires des insultes motsdoux, adopte une posture de surprise.

\section{Valeur des insultes " mots doux »}

Par valeur, il faut entendre le sens ou la signification profonde, la portée des insultes mot-doux dans les milieux cibles et par ricochet dans la société de manière générale. L'existence même d'un phénomène aussi abstrus et alambiqué que l'insulte mot-doux suffit pour ne pas dépriser ses valeurs qui sont culturelle, sociale et à la fois ludique et affective.

\subsection{Valeur culturelle}

Le culturel est en rapport avec la culture et s'oppose au " naturel ». Ainsi, les insultes mots-doux fonctionnent comme des acquis culturels hérités de nos ancêtres, et que nous devons préserver. Elles constituent une des meilleures manifestations des alliances et pactes multiséculaires tissés par nos aïeuls entre différents groupes ethniques. Ces pactes, dits de "non-agression », autorisent les groupes ethniques alliés, notamment leurs membres, à plaisanter entre eux. Cette plaisanterie outrepasse les limites de la plaisanterie ordinaire. Elle va au-delà, jusqu'à l'offense. Mais, l'offense résultant d'une telle plaisanterie ne devrait pas être prise comme un outrage. C'est justement ce qu'exploitent des insultes mots-doux, comme nous avons pu le montrer dans l'analyse dans (12 à 16) et (32 à 34).

Considérer l'offense faite dans le cadre de la plaisanterie de ce type est jugé attentatoire au pacte et doit être en principe sanctionné. Le caractère sacro-saint de ces pactes permet de consolider l'amour, la tolérance, la fraternité entre les groupes. Ces pactes constituent aussi un facteur de cohésion et de prévention de conflits. La preuve est donnée par l'exemple des insultes mots-doux qui, plutôt que de susciter la colère, le hérissement de leurs destinataires, au contraire les font sourire, parfois même au détriment de leur mauvaise humeur de l'instant. C'est pourquoi face aux insultes motsdoux, la réaction est plutôt calme, emprunte de convivialité. 


\subsection{Valeur ludique} nombre de ses faits et gestes. L'insulte mot-doux en est une manifestation. En dépit des nombreux drames issus de la cohabitation malheureuse entre les hommes, l'humain recherche sempiternellement la compagnie de ses semblables, avec qui mieux vivre. Or mieux vivre ne se limite pas uniquement à l'acquisition de biens, il suppose aussi partager, échanger, rire, s'épanouir... L'homme est fait pour vivre en communauté et en interaction avec ses semblables. Par ailleurs avec le développement de la société, la nécessité de vie en société s'accentue. Or justement cela n'est pas sans conséquences, $\mathrm{du}$ fait des antagonismes toujours latents. Les insultes mots-doux se présentent comme une solution pour prévenir la survenue d'antagonismes. Elles permettent de briser les barrières et de créer une ambiance emprunte de convivialité entre les hommes. Ce type de plaisanterie fonctionne comme un antidote du stress. Les insultes mots-doux en brisant les barrières, favorisent le rapprochement d'individus d'horizons divers non unis par quelque lien.

Les insultes mots-doux s'insèrent dans la lignée des insultes ludiques à l'instar du « gâtte-gâtte » dont elles s'inspirent du modèle de fonctionnement. Le " gâtte-gâtte » se présente comme un rituel de propos dévalorisants que des jeunes, organisés en deux équipes, échangent entre eux sous forme de joutes ludiques. Il fonctionne sur la base des règles à l'instar de tout jeu. La règle première consiste à proférer des insultes arbitraires qui n'ont aucun lien avec un quelconque "défaut» physique du destinataire. Ce qui importe dans ce jeu c'est l'escalade verbale.

vainqueur et un vaincu. Elles sont souvent motivées mais s'inscrivent dans une approche à la fois ludique et affective, généralement amusante, récréative, attachante ou divertissante. Elles s'échangent entre les jeunes, élèves, étudiants, apprentis, mais de manière générale entre jeunes ou promotionnaires d'âges. Le caractère ludique des insultes mots-doux réside dans le fait qu'elles n'agressent pas en réalité.

\subsection{Valeur sociale}

$\mathrm{Au}$ total, cette étude sur les "insultes mots-doux » a permis de montrer la connexité entre "insulte » et "insulte mot-doux » à travers une approche définitionnelle de chaque notion. Il s'agit de deux phénomènes qui sont très étroitement liés par nombre de leurs mécanismes de mise en œuvre. Toutefois, le fonctionnement de l'insulte qui consiste à vexer s'oppose à celui de l'insulte mot-doux qui produit l'effet inverse. Ainsi, des mots et expressions, généralement des adjectifs qualificatifs et des noms ou groupes nominaux, naturellement ou contextuellement violents, sont les constituants de l'insulte mot-doux avec une réception positive. Au demeurant le registre préférentiel de l'insulte mot-doux est familier voire relâché avec un mélange du nouchi et de langues du pays. L'insulte mot-doux se range en insulte mot-doux sexotype, tribale et personnelle. Loin d'être un phénomène banal, elle participe du rapprochement des populations et joue un rôle éminemment important sur les plans culturel et social. 


\section{BIBLIOGRAPHIE}

BUTLER, Judith, « Le pouvoir des mots. Politique du performatif », Paris, Éditions Amsterdam, (2004).

DERIVE, Jean, DERIVE, Marie Jo, « Processus de création et valeur d'emploi des insultes en français populaire de Côte-d'Ivoire ", dans " Les insultes : approches sémantiques et pragmatiques ", Langue française, $\mathrm{n}^{\circ}$ 144, Paris, Larousse, (2004), pp. 13-34.

DUBoIs, Jean etal., « Le dictionnaire de linguistique et des sciences du langage ", Paris, Larousse, 2012.

KERBRAT-ORECCHIONI, Catherine, « La Conversation », Paris, Éditions du Seuil, (1996).

KOFFI-LEZOU, Aimée-Danielle, « La violence verbale comme un exutoire. De la fonction sociale de l'insulte ", Signes, Discours et Sociétés [en ligne], 8, La force des mots : valeurs et violence dans les interactions verbales, 30 janvier 2012, Disponible sur Internet : http://www.revuesignes.info/document.php?id=2614. ISSN 1308-8378.

LAFOREST, Marty et VINCENT, Diane, « La qualification péjorative dans tous ses états ", Langue française, 144, (2004), pp. 59-81.

LAGORGETTE, Dominique, et LARRIVEE, Pierre, « Interprétation des insultes et relations de solidarité », Langue française 144 : (2004a), pp. 83-103.

LARGUÈCHE Evelyne, «L'effet injure, De la pragmatique à la psychanalyse », Collection Voix nouvelles en psychanalyse, Paris, PUF, (1983).

MOISE Claudine, « Espace public et fonction de l'insulte dans la violence verbale », dans Dominique Lagorgette (éd.), « Les insultes en français : de la recherche fondamentale à ses applications (linguistique, littérature, histoire, droit) », Chambéry, Université de Savoie, (2009), pp. 201-219.

\section{NOTES}

1. 1 Ce sont des personnes unies par des alliances interethniques. En Côte d'Ivoire, ce sont des pactes ancestraux de non-agression entre différentes ethnies qui permettent le maintien de la paix et de la cohésion sociale. Toutefois, ces pactes autorisent des « invectives » ou « offenses » à ne pas considérer comme agressives.

2. 2 Insulte célèbre, puisque prononcée publiquement par une dame députée, et grossière en langue malinké, qui désigne le sexe féminin.

\section{RÉSUMÉS}

Les insultes sont des faits et des actes qui rythment la vie communautaire. Elles surgissent généralement dans un environnement «confligène». Toutefois, l'on rencontre des cas 
« insolites » de leur manifestation, notamment dans des conversations entre des catégories ou classes sociales, professionnelles qui partagent une certaine connexité, où, loin d'offenser le destinataire et provoquer son hérissement, les insultes se font « mots-doux ».

Insults are speech acts that punctuate social life. They arise mostly in a conflicting environment. However, an anusual case of insult can occur especially in conversations between some socioprofessional categories that share a certain connection. These types of insult do not cause any offence that may provoke the addressee's anger, because it is done through "gentle words".

INDEX

Mots-clés : conversation, espace public, insultes, insultes mots-doux, mots-doux

Keywords : conversation, public places, insult, gentle-words insults, gentle-words

\section{AUTEUR}

\section{KOUAKOU KOUMAN FODJO}

École Normale Supérieure Abidjan, Côte d'Ivoire 